\title{
Knowledge, opinions and attitudes of Italian mothers towards HPV vaccination and Pap test
}

\author{
Giuseppe La Torre ${ }^{1}$, Elisabetta De Vito ${ }^{2}$, Maria Giovanna Ficarra ${ }^{3}$, Alberto Firenze ${ }^{4}$, Pasquale Gregorio ${ }^{5}$, Silvia Miccoli ${ }^{1}$, \\ Guglielmo Giraldi ${ }^{1}$, Brigid Unim ${ }^{1}$, Giulio De Belvis ${ }^{3}$, Antonio Boccia ${ }^{1}$, HPV Collaborative Group: Rosella Saulle ${ }^{1}$, Leda \\ Semyonov ${ }^{1}$, Maria Ferrara ${ }^{2}$, Elisa Langiano ${ }^{2}$, Silvio Capizzi ${ }^{3}$, Rosaria Nardella ${ }^{3}$, Maria Grazia Laura Marsala ${ }^{4}$, Valentina \\ Bonanno $^{4}$, Clara Ferrara ${ }^{4}$, Enrica Guidi ${ }^{5}$, Mauro Bergamini ${ }^{5}$, Silvia Lupi ${ }^{5}$
}

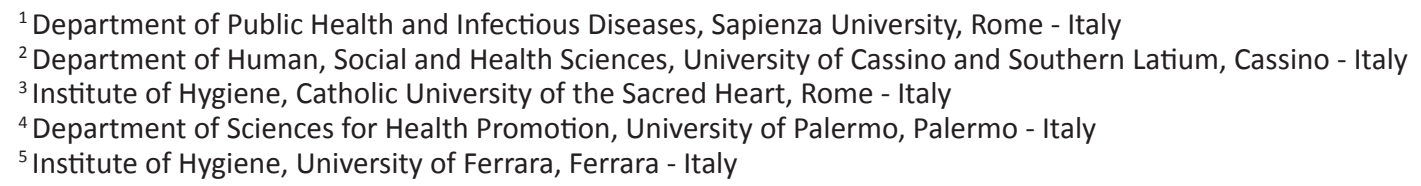

\begin{abstract}
Aim: This study evaluated the knowledge and attitudes of Italian mothers - whose daughters had been vaccinated in 2012 - towards primary (anti-HPV vaccination) and secondary (Pap test screening) cervical cancer prevention, as well as sources of information and mother-daughter communication on health issues.

Methods: The survey - part of a multicenter study carried out in 4 Italian cities (Ferrara, Rome, Cassino and Palermo) - was conducted through self-administered questionnaires. The first univariate analysis evaluated differences between mothers of under-18s and over-18s relative to knowledge and attitudes on HPV vaccination and Pap test. The second univariate analysis evaluated differences between the 2 groups of mothers and possible geographical variations regarding the sources of information on HPV and Pap test.

Results: The sample proved knowledgeable about the correlation between HPV and cervical cancer (>85\%) but less aware of other HPV-related diseases. HPV vaccination should be administered before first sexual intercourse according to mothers of over-18s, and to 14 - to 17 -year-olds according to mothers of under-18s. Up to $88 \%$ of mothers of under- $18 \mathrm{~s}$ and $80 \%$ of mothers of over- $18 \mathrm{~s}$ declared that the vaccine should be given free of charge. More mothers of under-18s consulted a general practitioner (GP) or gynecologist before deciding to vaccinate their daughters. Mothers of under-18s received information on HPV vaccination mainly from GPs and gynecologists, while mothers of over-18s were informed through TV and books/journals. Over $80 \%$ of the sample declared satisfaction with the information received from their gynecologist during the Pap test.

Conclusions: The findings provide useful information for the development of effective public health interventions that may help improve acceptance of HPV vaccination among mothers.
\end{abstract}

Keywords: Human papillomavirus, Mothers, Pap-test, Sexually transmitted infections, Vaccination

\section{Introduction}

Human papillomavirus (HPV) is an important and commonly studied virus responsible for sexually transmitted infections (STIs). The high-risk HPV types cause invasive cervical cancer, which is the second most common cancer in women

\author{
Accepted: February 3, 2015 \\ Published online: \\ Corresponding author: \\ Prof. Giuseppe La Torre \\ Department of Public health and \\ Infectious Diseases Sapienza University of Rome \\ Piazzale Aldo Moro 5 \\ 00185 Roma, Italy \\ giuseppe.latorre@uniroma1.it
}

with an estimated 530,000 new cases every year (1). Risk factors for HPV infection and development of cervical cancer have been identified: early first sexual intercourse, multiple sexual partners, immune suppression, and tobacco use. Other strains of the virus cause less serious health problems (2-5).

The prevalence of any type of HPV infection in Italy ranges between $7 \%$ and $16 \%$ among women aged $17-70$ years; the prevalence of genital HPV infection is higher among sexually active Italian women under 25 years of age and amounts to $24.1 \%(6,7)$.

HPV vaccination is recognized as a valid method of prevention as it is safe and offers long-lasting protection (up to 8 years). Also, it is particularly effective before first sexual intercourse, hence before exposure to the viruses. Trivalent and quadrivalent HPV vaccines were introduced in the vaccination schedule of 18 European countries by 2010. In Italy, 
vaccination is recommended and offered free of charge to all girls at 12 years of age; the vaccine can be administered to women from 9 through 45 years of age in 3 doses. By June 2012, the national coverage for girls born between 1997 and 1999 was about $70 \%$ (8).

In the USA, the Centers for Disease Control and Prevention (CDC) and the American Academy of Pediatrics recommend HPV vaccination of both girls and boys at ages 11 or 12 years. Vaccination is also recommended for females up to 26 years and males up to 21 years of age who have not completed the vaccine series, and to immunocompromised people aged 22-26 years $(9,10)$. HPV vaccination is recommended to both genders also in Canada (ages 9-26), Australia, and New Zealand (9-26 years for females and 9-15 years for males), but not yet in Italy $(11,12)$.

Vaccinated females will still need regular cervical cancer screening (Pap test) beginning at age 21, since vaccination protects against most but not all of the HPV types that cause cervical cancer. In addition, protective sexual behavior (i.e., condom use) is also necessary since the vaccine will not prevent other STIs (9).

The present study was developed considering that HPV vaccination campaigns for young women have been carried out in all Italian local health units (LHUs). The aim of the analysis was to evaluate the knowledge, opinions and attitudes of Italian mothers - whose daughters had been vaccinated in 2012 - towards primary (anti-HPV vaccination) and secondary (Pap test screening) cervical cancer prevention, as well as sources of information and mother-daughter communication about health issues.

\section{Methods}

The present work is part of a multicenter study called PRIN, Programmi di Ricerca di Rilevante Interesse Nazionale (Research Projects of National Interest), carried out in 4 cities (Ferrara, Rome, Cassino and Palermo) by the Sapienza University of Rome, the Catholic University of the Sacred Heart of Rome, and the Universities of Palermo, Cassino and Ferrara.

The regions of the participating universities were selected for being representative of the different geographical areas in Italy relative to knowledge, attitudes and opinions towards HPV vaccination, Pap test screening, and reliable sources of information about those issues. In particular, Ferrara is in a northern region, Rome and Cassino are in central Italy, and Palermo is in southern Italy.

The study participants were mothers who accompanied their daughters (under-18s and over-18s) to LHUs for HPV vaccination and were interviewed in the waiting rooms. Informed consent was obtained from the mothers and the study protocol was approved by the ethics committee of Sapienza University of Rome. The analysis regarding the young women who underwent vaccination was carried out in $2013(13,14)$.

\section{The questionnaire}

The survey was conducted through a questionnaire composed of 3 sections. The first part accounted for 9 questions investigating knowledge, opinions, attitudes and sources of information about primary cervical cancer prevention
(anti-HPV vaccination) and 3 questions about motherdaughter communication. The questionnaire administered to mothers of under-18s included 2 additional questions to evaluate the mother's knowledge about HPV vaccination before receiving the invitation letter from the LHU, and to ascertain who was the first in the family to accept the invitation for the vaccination.

The second part was composed of 6 questions relative to knowledge, opinions, attitudes and sources of information about secondary cervical cancer prevention (Pap test screening). The third section dealt with sociodemographic data including age, nationality, region of residence, religion, educational level, and occupational status (6 questions).

\section{Statistical analysis}

Data were stored using the database software DB (version IV) C. Wayne Ratliff, 1979 and processed with the statistical software SPSS 19.0 for Windows (SPSS Inc., Chicago, IL, USA), with the level of significance set at $p<0.05$. Descriptive statistics were calculated for quantitative variables (mean and standard deviation [SD]) and qualitative variables (frequency distribution). The first univariate analysis evaluated possible differences between mothers of under-18s and over-18s relative to knowledge, attitudes and opinions about HPV vaccination, Pap test screening, and other related issues. The second univariate analysis evaluated differences between the 2 groups of mothers and possible geographical variations regarding the sources of information about HPV and Pap test. The chi-square test was used in both analyses.

\section{Results}

\section{Sociodemographic characteristics}

The sample was composed of 444 mothers of young women who underwent HPV vaccination during the study period. The mothers had a mean age of 44.87 years $(S D=5.97)$ and up to $79 \%$ resided in central and southern Italian regions. The majority of the sample was Italian (97\%) and affirmed to be catholic (86.4\%). The educational level was senior high school in $59 \%$ of mothers and academic in $35.7 \%$; more than half of the mothers worked (65\%).

\section{Knowledge, opinions and attitudes towards HPV vaccination, Pap test and other sexually related issues}

With regard to the questions addressed only to mothers of under-18s, it was found that $83.8 \%$ knew about HPV vaccination before receiving the invitation letter from their LHU and $60 \%$ were the first to accept the invitation with respect to other members of the family. In fact, only $6.8 \%$ of daughters aged under 18 years were the first to accept it, and $19.4 \%$ accepted the invitation together with their mothers (data not presented in Tab. I).

The sample demonstrated good knowledge about the correlation between HPV and cervical cancer (over 85\%), but was less aware of other diseases caused by HPV viruses (Tab. I). More mothers of under-18s knew that a Pap test is still required after HPV vaccination compared with mothers of 
TABLE I - Knowledge, opinions and attitudes of mothers towards HPV vaccination

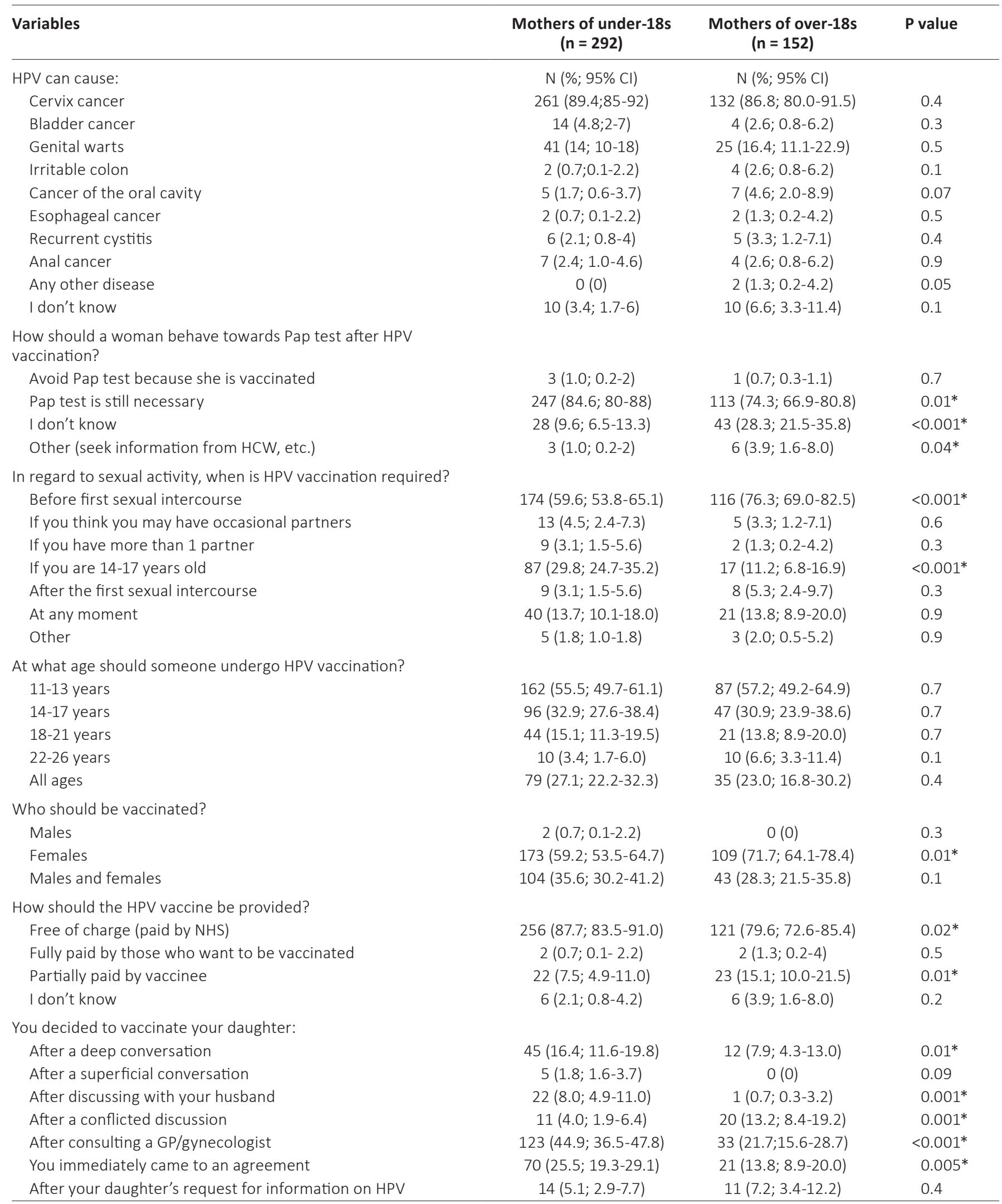

$\mathrm{HPV}=$ human papillomavirus; $\mathrm{Cl}=$ confidence interval; $\mathrm{HCW}=$ healthcare worker; NHS = National Health Service; GP = general practitioner . ${ }^{*} p<0.05$. 
over-18s ( $84.6 \%$ vs. $74.3 \% ; p=0.01)$. Some participants did not know at all how a woman should behave after undergoing the vaccination ( $28.3 \%$ of mothers of over- 18 s vs. $9.6 \%$ of mothers of under-18s; $\mathrm{p}<0.001)$ and very few mothers $(3.9 \%$ and $1 \%$, respectively; $p=0.04$ ) declared they would seek information from a healthcare worker.

HPV vaccination should be administered before the first sexual intercourse according to mothers of over-18s $(p<0.01)$, and to 14- to 17-year-olds according to mothers of under-18s $(\mathrm{p}<0.01)$.

More than $50 \%$ of the sample stated that the target age for HPV vaccination should be 11-13 years. The majority of the sample also found that vaccination should include the female gender only $(59.2 \%$ of mothers of under- $18 \mathrm{~s}$ and $71.7 \%$ of mothers of over-18s; $p=0.01$ ). In addition, the vaccine should be given free of charge according to $87.7 \%$ of mothers of under-18s and $79.6 \%$ of mothers of over- $18 \mathrm{~s} ; \mathrm{p}=0.02$ ) or be partially charged to the vaccinee $(7.5 \%$ and $15 \%$, respectively; $p=0.01$ ).

More mothers of under-18s consulted a general practitioner (GP) or gynecologist prior to the decision to vaccinate their daughters $(p<0.001)$; came immediately to an agreement $(p=0.005)$; decided after a deep conversation with their daughters ( $p=0.01)$, or after discussing with their husbands $(p=0.001)$. Conversely, more mothers of over-18s had a conflicted discussion with their daughters $(p=0.001)$.

The knowledge, opinions and attitudes of the participants toward the Pap test are summarized in Table II. About $85 \%$ of mothers of under- $18 \mathrm{~s}$ and $46 \%$ of mothers of over18 s claimed to know what a Pap test is $(p<0.001)$, and more than $85 \%$ of the sample had undergone the test themselves. More mothers of over-18s had participated in a cervical cancer screening program organized by their LHU $(p<0.001)$, but declared to be less satisfied with the program than mothers of under-18s $(p<0.001)$.

According to our data, mothers of under-18s had a better relationship with their daughters: $80.5 \%$ affirmed having a confidential/intimate relationship $(p<0.001)$, while only $5 \%$ considered their relationship formal $(p<0.001)$; over $70 \%$ talked about sexually related topics $(p<0.001)$ such as menstruation $(p<0.001)$, sexually transmitted diseases $(p=0.001)$, and contraception $(p=0.003)$.

\section{Sources of information}

As shown in Table III, mothers of under-18s received information on HPV vaccination mainly from their GPs $(p=0.005)$ or gynecologists $(p=0.004)$, while mothers of over-18s were informed through TV $(p=0.001)$ and books/journals $(p=0.04)$. Geographically, GPs were the main source of information in central regions, TV in southern areas, and books/journals in northern Italy $(p<0.001)$.

Differences emerged also with regard to the daughters' main source of information, which included their mothers $(p<0.001)$, LHUs $(p=0.006)$ and pediatricians $(p=0.02)$ for girls under 18. Geographically, daughters were mostly informed by their mothers $(p<0.001)$, GPs $(p=0.04)$, and gynecologists $(p=0.03)$ in central Italy. Important sources particularly in southern regions were friends $(p<0.001), \operatorname{TV}(p=0.005)$, and websites $(p=0.01)$.
Over $80 \%$ of the mothers of the sample declared they were satisfied with the information received from their gynecologist during the Pap test, mostly in central Italy (89\%). Nevertheless, they did not receive specific information about HPV infections during the medical visit $(52 \%$ of mothers of under-18s and $62 \%$ of mothers of over-18s), especially those residing in northern regions.

\section{Discussion}

According to the most recent available data, the cervical cancer incidence and mortality among Italian women in 2005 were $1.6 \%$ and $0.6 \%$, respectively (15). Preventive measures against cervical cancer are essentially 2 : cervical cancer screening with the Pap test and anti-HPV vaccination (16).

In agreement with our research, most studies (17-19) report high rates of parental acceptance of HPV vaccination: we found $60 \%$ of acceptance toward vaccine administration among mothers. Our sample demonstrated good knowledge about the relation between HPV and cancer, which was not observed in the study by Barnack et al (19).

Numerous variables may influence the decision of parents to vaccinate their children. Parental consent is generally required for medical interventions to adolescents younger than 18 years, so parents' perceptions about sexuality, vaccination and STIs may play an important role in determining the uptake of the HPV vaccine among adolescent girls (20).

Physicians have an important role in cancer prevention and thus in providing evidence-based recommendations to parents, especially the hesitant ones (21). Family physicians are the principal source of information for most mothers (22), and well-informed mothers are more prone to having their daughters vaccinated (23). We found that the majority of mothers decided to vaccinate their daughters after consulting a general practitioner or gynecologist.

Regarding the age at which girls should be vaccinated, the majority of respondents in a study considered young age to be appropriate for HPV vaccination, in particular before first sexual intercourse (24). Consistent with these findings, we found that about $60 \%$ of mothers of under- $18 \mathrm{~s}$ and $76 \%$ of mothers of over-18s believed vaccination should be done before sexual activity.

Most mothers identified the ages from 11 to 13 as optimal for HPV vaccination. This is in contrast with the study by Jaccard et al (25), where mothers preferred vaccinating their daughters at a later age, probably because some parents are convinced that HPV vaccination leads to sexual disinhibition.

At the age of 13.4 and 11.8 years, respectively, $2.0 \%$ and $0.5 \%$ of women have had their first sexual experience; about $50 \%$ of women contract genital HPV infections within the first 2 years of sexual activity (26). Family physicians and public health professionals should address these issues by underlining that the highest rate of protection is obtained when the vaccine is administered before the first sexual experience. Hence, vaccinating 10 - to 12 -year-olds can protect the majority of girls. Several studies have demonstrated the importance of practitioner recommendation in HPV vaccine uptake for adolescents as well as in health promotion interventions $(24,27)$. 
TABLE II - Knowledge, opinions and attitudes of mothers towards Pap test and other sexually related issues

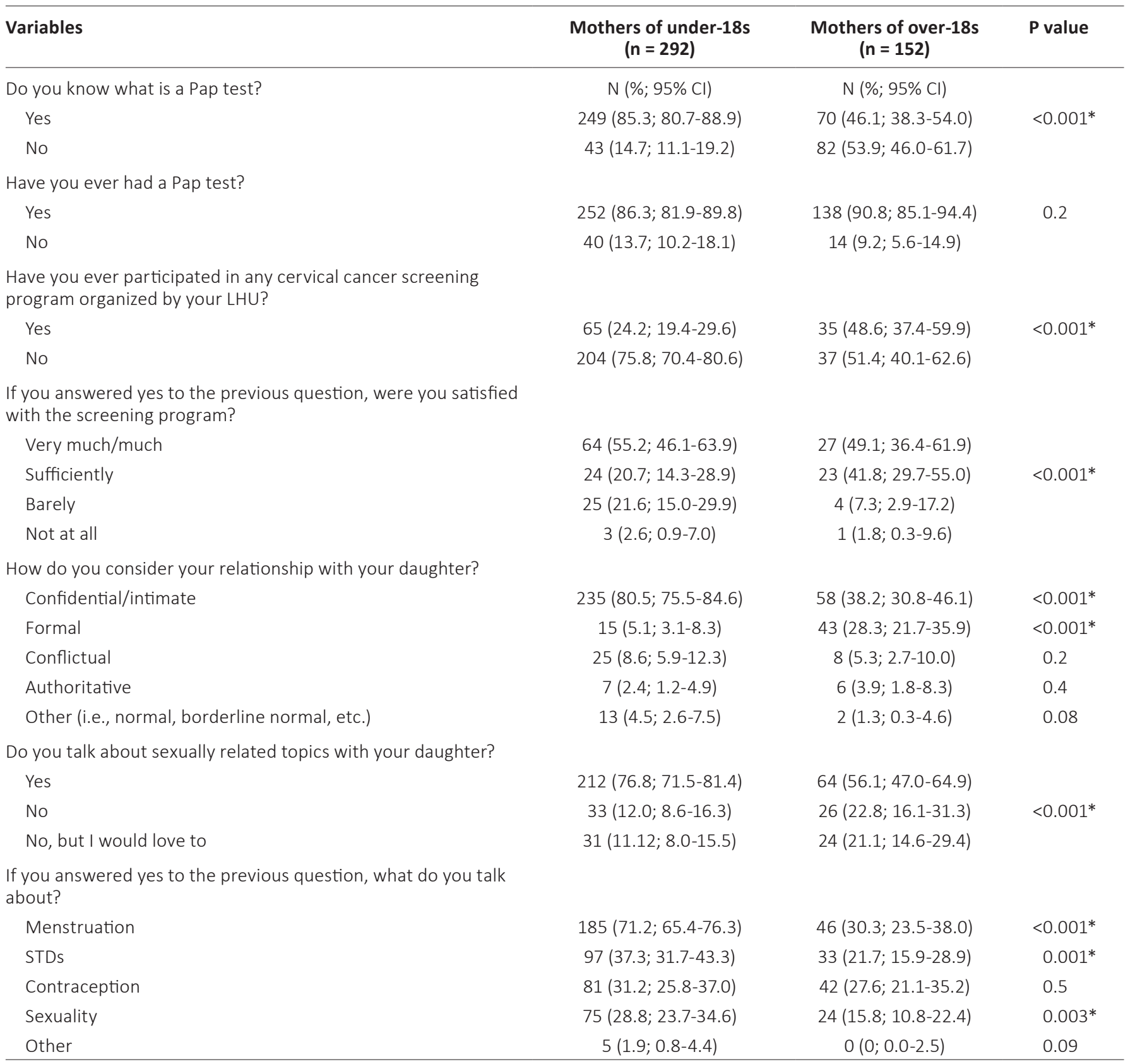

$\mathrm{Cl}=$ confidence interval; $\mathrm{LHU}=$ local health unit; STD = sexually transmitted disease. ${ }^{*} p<0.05$.

In our sample, most mothers (85\%) were aware of the Pap test and had it done themselves. This practice may have encouraged them to accept screening for their daughters as well; in fact, a study reported that women who participated in cervical cancer screening programs were more prone to vaccinating their daughters than women who had never had a Pap test (28). Our findings confirm the evidence for the key role of parents in orienting the decision to vaccinate their daughters and strengthen the necessity of vaccination programs to tackle parents beliefs about sexual disinhibition and promote the importance of vaccination (29).
In the US, the aim of Healthy People 2020 is to achieve high population vaccination coverage, but unfortunately only part of the population is willing to consider HPV vaccination (30). In this sense, the source of information can play a crucial role. Our results showed that over $80 \%$ of mothers were satisfied with the information received from their gynecologist during the Pap test; the satisfaction rate was highest among mothers from central Italy (89\%). Furthermore, we found that websites were an important source of information, especially in the south of Italy. These findings are consistent with other studies $(14,31)$ showing that the media play a key role in 


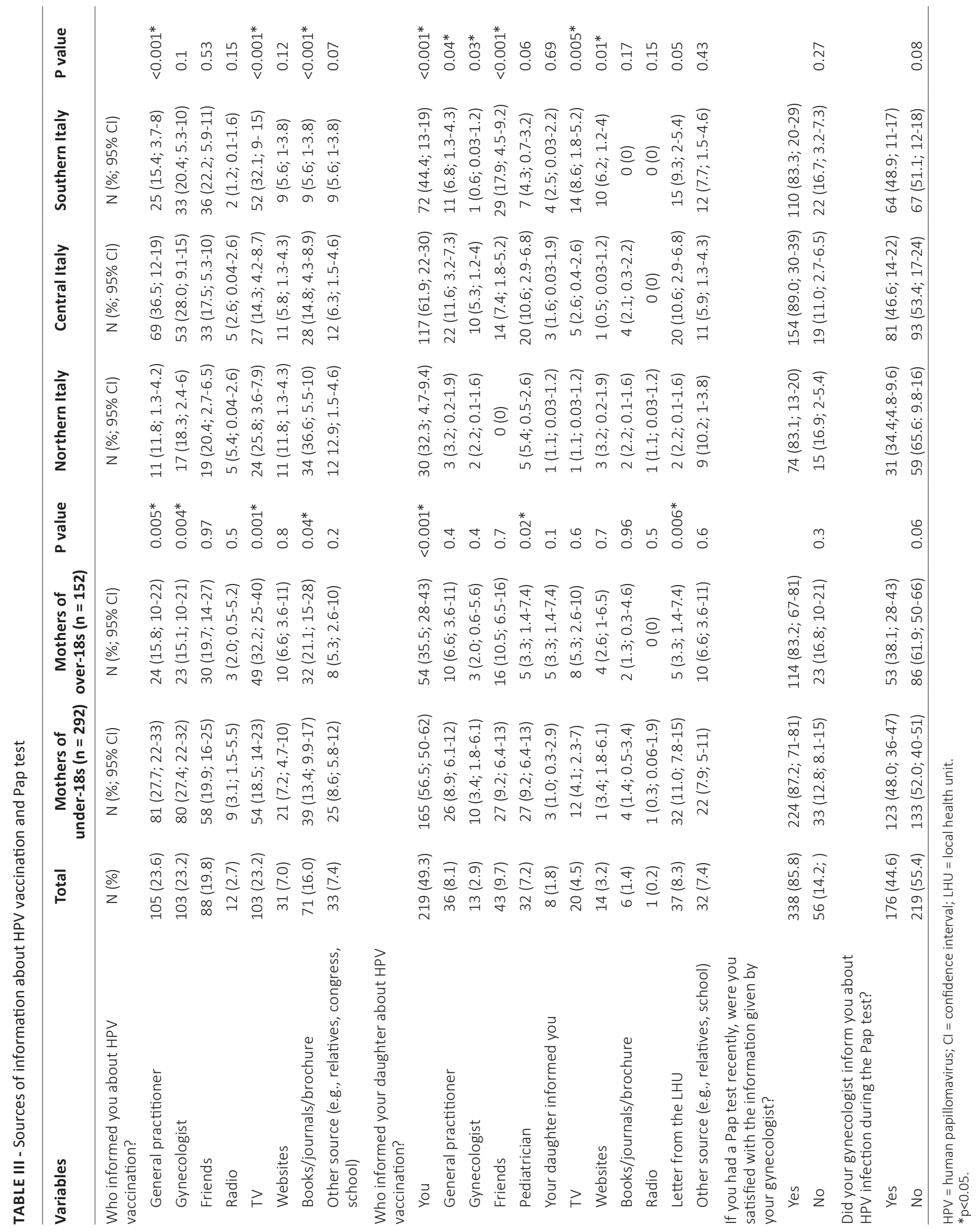


health matters; in fact, about $47 \%$ of Europeans seek health information online.

The major strength of this study is that it provides information on HPV prevention among mothers of young Italian women. These data may be considered as nationally representative because the study analyzed women from northern, central and southern Italy.

However, our study has some limitations. We analyzed a sample of mothers attending health facilities with their daughters, and the results may have been different in other populations or settings, although we performed a multicenter study. Mothers who participate in these type of studies could be more favorable toward vaccination than those who refuse to participate or those who do not accompany their daughters when seeing a physician (32). Also, due to the cross-sectional design it is difficult to establish whether the knowledge about the HPV vaccine was related to information given during the vaccination and whether it influenced the decision to undergo vaccination (33).

In our sample, the majority of mothers were in favor of HPV vaccination at an early age. This is important even if the sample might not have been representative of the whole Italian population. The present study provides useful information for the development of effective public health interventions that may improve parental acceptance of HPV vaccination and therefore vaccination coverage in adolescents.

\section{Disclosures}

Financial support: This research was funded by Ministerial grant PRIN 2008 (Ministry of Education, University and Research)

Conflict of interest: The authors declare there is no conflict of interests

\section{References}

1. Arbyn $M$, Castellsagué $X$, de Sanjosé $S$, et al. Worldwide burden of cervical cancer in 2008. Ann Oncol. 2011;22(12):2675-2686.

2. World Health Organization. Human papillomavirus (HPV) and cervical cancer. Fact sheet No. 380; 2013. Available at: http:// www.who.int/mediacentre/factsheets/fs380/en/. Accessed April 21, 2014.

3. Dunne EF, Markowitz LE. Genital human papillomavirus infection. Clin Infect Dis. 2006;43(5):624-629.

4. American Cancer Society. Cervical cancer overview. Available at: www.cancer.org/Cancer/CervicalCancer/OverviewGuide/index. Accessed April 29, 2014.

5. Richardson H, Kelsall G, Tellier $P$, et al. The natural history of type-specific human papillomavirus infections in female university students. Cancer Epidemiol Biomarkers Prev. 2003;12 (6):485-490.

6. Epicentro ISS. Infezioni da HPV in Italia. Available at: http://www. epicentro.iss.it/problemi/hpv/epid.asp. Accessed May 3, 2014.

7. Ammatuna P, Giovannelli L, Matranga D, Ciriminna S, Perino A. Prevalence of genital human papilloma virus infection and genotypes among young women in Sicily, South Italy. Cancer Epidemiol Biomarkers Prev. 2008;17(8):2002-2006.

8. Società Italiana di Igiene (SiTi). VaccinarSì: vaccino anti-HPV (papillomavirus umano). Available at: http://www.vaccinarsi.org/vaccini-disponibili/vaccino-anti-hpv.html. Accessed May 21, 2014.

9. Centers for Disease Control and Prevention (CDC). HPV vaccine information for clinicians - Fact sheet. Available at: http:// www.cdc.gov/std/hpv/stdfact-hpv-vaccine-hcp.htm. Accessed April 28, 2014.
10. Giraldi G, De Luca d'Alessandro E. The HPV infection in males: an update. Ann Ig. 2012;24(6):497-506.

11. PublicHealthAgencyofCanada. Humanpapillomavirus(HPV)preventionandHPVvaccines.Availableat:http://www.phac-aspc.gc. ca/std-mts/hpv-vph/hpv-vph-vaccine-eng.php). Accessed May 15, 2014.

12. The Royal Australian and New Zealand College of Obstetricians and Gynaecologists. College Statement (C-Gyn 18): Guidelines for HPV vaccine. Available at: http://www.ranzcog.edu.au/ doc/guidelines-for-hpv-vaccine.html. Accessed May 20, 2014.

13. Saulle R, Miccoli S, Unim B, et al. Validation of a questionnaire for young women to assess knowledge, attitudes and behaviors towards cervical screening and vaccination against HPV in Italy. Epidemiology Biostatistics and Public Health. 2014;11 (2):e8913-1- e8913-12.

14. La Torre G, De Vito E, Ficarra MG, Firenze A, Gregorio P, Boccia A; HPV Collaborative Group. Is there a lack of information on HPV vaccination given by health professionals to young women? Vaccine. 2013;31(42):4710-4713.

15. AIRTUM Working Group. I tumori in Italia - Rapporto AIRTUM 2009. I trend dei tumori negli anni 2000 (dati 1998-2005). Epidemiologia e Prevenzione. 2009;33(4-5 Suppl 1):1-167.

16. Muñoz N, Bosch FX, de Sanjosé S, et al; International Agency for Research on Cancer Multicenter Cervical Cancer Study Group. Epidemiologic classification of human papillomavirus types associated with cervical cancer. N Engl J Med. 2003;348 (6):518-527.

17. Das A, Madhwapathi V, Davies P, et al. Knowledge and acceptability of the HPV vaccine by school children and their parents in Birmingham. Vaccine. 2010;28(6):1440-1446.

18. Yeganeh N, Curtis D, Kuo A. Factors influencing HPV vaccination status in a Latino population; and parental attitudes towards vaccine mandates. Vaccine. 2010;28(25):4186-4191.

19. Barnack JL, Reddy DM, Swain C. Predictors of parents' willingness to vaccinate for human papillomavirus and physicians' intentions to recommend the vaccine. Womens Health Issues. 2010;20(1):28-34.

20. Zimet GD, Perkins SM, Sturm LA, Bair RM, Juliar BE, Mays RM. Predictors of STI vaccine acceptability among parents and their adolescent children. J Adolesc Health. 2005;37(3):179-186.

21. Ogilvie G, Anderson M, Marra F, et al. A population-based evaluation of a publicly funded, school-based HPV vaccine program in British Columbia, Canada: parental factors associated with HPV vaccine receipt. PLoS Med. 2010;7(5):e1000270.

22. Freed GL, Clark SJ, Butchart AT, Singer DC, Davis MM. Sources and perceived credibility of vaccine-safety information for parents. Pediatrics. 2011;127(Suppl 1):S107-S112.

23. Litton AG, Desmond RA, Gilliland J, Huh WK, Franklin FA. Factors associated with intention to vaccinate a daughter against HPV: a statewide survey in Alabama. J Pediatr Adolesc Gynecol. 2011;24(3):166-171.

24. Lenselink $\mathrm{CH}$, Gerrits MM, Melchers WJ, Massuger LF, van Hamont D, Bekkers RL. Parental acceptance of human papillomavirus vaccines. Eur J Obstet Gynecol Reprod Biol. 2008;137 (1):103-107.

25. Jaccard J, Dittus PJ, Gordon VV. Parent-adolescent congruency in reports of adolescent sexual behavior and in communications about sexual behavior. Child Dev. 1998;69(1):247-261.

26. Del Prete G, Giraldi G, Miccoli S, et al. Adolescents' affectivity and sexuality: a randomized trial of the efficacy of a school health promotion intervention in a primary school. Ig Sanita Pubbl. 2012;68(6):821-840.

27. Kjaer SK, Chackerian B, van den Brule AJ, et al. High-risk human papillomavirus is sexually transmitted: evidence from a followup study of virgins starting sexual activity (intercourse). Cancer Epidemiol Biomarkers Prev. 2001;10(2):101-106. 
28. Spencer Nee Pilkington AM, Brabin L, Verma A, Roberts SA. Mothers' screening histories influence daughters' vaccination uptake: an analysis of linked cervical screening and human papillomavirus vaccination records in the North West of England. Eur J Cancer. 2013;49(6):1264-1272.

29. Allen JD, Othus MK, Shelton RC, et al. Parental decision making about the HPV vaccine. Cancer Epidemiol Biomarkers Prev. 2010;19(9):2187-2198.

30. Harper DM, Irons BB, Alexander NM, et al. Quantifying the decisional satisfaction to accept or reject the Human Papillomavirus (HPV) vaccine: a preference for cervical cancer prevention. PLoS ONE. 2014;9(2):e88493.
31. Siliquini $R$, Ceruti $M$, Lovato $E$, et al. Surfing the internet for health information: an italian survey on use and population choices. BMC Med Inform Decis Mak. 2011;11(1):21.

32. Perkins RB, Apte $G$, Marquez $C$, et al. Factors affecting human papillomavirus vaccine use among White, Black and Latino parents of sons. Pediatr Infect Dis J. 2013;32(1): e38-e44.

33. Haesebaert J, Lutringer-Magnin D, Kalecinski J, et al. French women's knowledge of and attitudes towards cervical cancer prevention and the acceptability of HPV vaccination among those with 14-18 year old daughters: a quantitative-qualitative study. BMC Public Health. 2012;12:1034. 